Schooler, N. R., Keith, S. J., Severe, J. B., et al (1997) Relapse and rehospitalisation during maintenance treatment of schizophrenia. Archives of General Psychiatry, 54, 453-463.

I. R. H. Falloon Department of Psychiatry and Behavioural Science, Faculty of Medical and Health Sciences, University of Auckland, Private Bag 92019, Auckland, New Zealand

Authors' reply: We appreciate Professor Falloon's comments about our paper (Bellack et al, 2000), as well as his thoughts about the broader Treatment Strategies in Schizophrenia (TSS) project from which we drew the subjects of our paper. He is certainly correct that the study was not designed to systematically dismantle his behavioural family therapy (referred to as 'applied family management' in our study), and that we did not conduct a formal economic analysis of the outcomes.

Most of his comments refer to the parent TSS study, its design, its conduct and the interpretation of its results, and go substantially beyond the relatively limited questions that we addressed in our paper. The goal of our article was to examine the effects of family treatments on communication and whether changes in communication mediated patient outcomes. The TSS study compared behavioural family treatment with a less-structured family support programme. In Bellack et al (2000) we reported that there were no differences between the two family treatments in communication or problem-solving, and changes in communication that may have occurred did not mediate outcomes of interest, including any difference in rehospitalisation.

The TSS study was, as Professor Falloon has written (Falloon et al, 1996), designed to compare two family treatments based on common assumptions and common principles. Applied family treatment (AFM) was based on the behavioural family therapy developed by Falloon and "differed from Supportive Family Treatment in intensity and in the site of delivery of treatment ... Further, AFM has a behavioral focus, with the intent of providing specific training in communication and problem solving" (Falloon et al, 1996, page 47). Both family treatments included parallel family support groups, and the two treatments did not differ in their orientation, as Professor Falloon now suggests. Stress management was a feature of the two family interventions, but they were not conceptualised that narrowly. Falloon's treatment (Falloon et al, 1996), in particular, was viewed as a comprehensive intervention that included case management and a multi-factorial educational component designed to modify patterns of communication within the family. He reports that the problem-solving component "seldom requires more than 5 hours of teaching", yet his programme required 13 weekly sessions in the home followed by 13 bi-weekly sessions and then monthly sessions for up to an additional 6 months. Neither the data nor our own clinical observations support the thesis that most families are able to learn the targeted skills at all, let alone in 5 hours of training. In regard to cost benefit, it should be noted that there was no demonstrable benefit from this extensive training and the cost of more than a year of home visits would be prohibitive in most clinical settings: a statistical economic analysis would be redundant with this self-evident finding.

Professor Falloon makes two other assertions with which we disagree. First, he indicates that the monthly educational groups and the behavioural training were incompatible and that therapists "confused the two approaches". On the contrary, the two approaches were designed to support one another by providing a common model of the illness to families in two different forums with the added benefit of multifamily support and sharing. Moreover, Professor Falloon provided quality control for the home visits but had no ongoing role in oversight of the multi-family support groups, so it is difficult to understand how he determined the existence of this putative confusion on the part of therapists.

Second, Professor Falloon notes that there was a difference in rehospitalisation between the two family treatments over 2 years under "optimal" medication conditions. The numbers cited are selected from a large analysis that tested the effects of the family treatment comparison as well as of medication condition. There was neither a statistically significant main effect of family treatment nor an interaction of family treatment and medication. The difference he cites is thus not appropriately described as "not quite" statistically significant. The clear and overarching conclusions to be drawn from the entire data set is that Falloon's behavioural family therapy did not produce any differential benefit to family members or patients, despite its high cost. We strongly support clinical and humanistic goals of improving the quality of interactions in families with a child suffering from schizophrenia, and of reducing stress experienced by family members and patients. However, we also believe that the TSS data provide a convincing argument that the behavioural treatment approach is not a useful or effective strategy for most families.

Bellack, A. S., Haas, G. L., Schooler, N. R., et al (2000) Effects of behavioural family management on family communication and patient outcome in schizophrenia. British Journal of Psychiatry, 177, 434-439.

Falloon, I. R. H., McGill, C. W., Matthews, S. M., et al (1996) Family treatment for schizophrenia: the design and research application of therapist training models. Journal of Psychotherapy Practice and Research, 5, 45-56.

A. S. Bellack VA Capitol Health Care Network and University of Maryland, School of Medicine, Center for the Behavioral Treatment of Schizophrenia, 737 West Lombard Street, Suite 55I, Baltimore, MD 2120I, USA

N. R. Schooler Long Island Jewish Medical Center, New York, USA

\section{Psychological therapies in anorexia nervosa}

It is heartening to see trials of therapy for anorexia nervosa, the most lethal of psychiatric illnesses and the cinderella of research. Dare et al (2001) have shown that over a year it is possible to effectively treat a severely ill group of young adults with poor prognostic features, and to do so on an out-patient basis.

I am surprised, though, that they feel able to conclude that "specialised psychotherapies are more effective than routine treatment". The two therapies which came out 'top' (family therapy and focal psychoanalytic psychotherapy) were given by the same three highly experienced therapists, and the next best therapy (cognitive-analytic therapy) was given by trained specialists in eating disorders, whereas 'routine treatment' was provided by junior psychiatrists on 6-month rotations who had to hand over to colleagues during the year of patient contract.

Certainly, confidence in at least one model of therapy is an important component of an experienced therapist's effectiveness, but for me the clearest implication of the study is that patients who suffer from this chronic condition do best with 